\title{
PERSPECTIVE
}

\section{Options for Meaningful Engagement in Clinical Research for Busy Frontline Clinicians}

\author{
Karen M. Goldstein, MD, MSPH ${ }^{1,2,3}$ (D), Jennifer M. Gierisch, $P h D, M P H^{1,2,4}$, \\ Matthew Tucker, BA ${ }^{7}$, John W. Williams Jr, MD, MHSc ${ }^{1,2}$, Rowena J. Dolor, MD, MHS' , and \\ Wendy Henderson, $M D^{1,2}$ \\ 'Durham Veterans Affairs Health Care System, Durham, NC, USA; ${ }^{2}$ Department of Medicine, Division of General Internal Medicine, Duke University \\ School of Medicine, Durham, NC, USA; ${ }^{3}$ Durham Center of Innovation to Accelerate Discovery and Practice Transformation, Veterans Affairs \\ Health Services Research and Development, Durham, NC, USA; ${ }^{4}$ Department of Population Health Sciences, Duke University, Durham, NC, USA.
}

In order for health care innovations to be effective and actionable, they must align with the needs and practice patterns of those delivering care at the bedside. While research has started to incorporate the patient voice, it has yet to fully invest in the expertise of frontline clinicians. Frontline clinicians carry a wealth of clinical knowledge and the lived experience of providing realworld medical care that the research community seeks to improve. We consider options for clinicians as research stakeholders along a continuum of engagement as outlined by the UCSF Clinical and Translational Science Institute from minimal to supportive to participatory. In order to make an effective value proposition to support reallocation of clinician time to research engagement, we advocate evaluating the impact of clinicians as stakeholders at both the process level (e.g., clinician satisfaction, study recruitment rates) and endpoint level (e.g., clinical outcomes). Investing in clinicians as research stakeholders can offer benefits for the individual, health system, and population by increasing the generalizability, adoption, and sustainability of effective interventions.

$\mathrm{J}$ Gen Intern Med 36(7):2100-4

DOI: $10.1007 / \mathrm{s} 11606-020-06587-3$

(c) This is a U.S. government work and not under copyright protection in the U.S.; foreign copyright protection may apply 2021

\section{INTRODUCTION}

Health care innovations can be ineffectual and irrelevant without input from those delivering health care at the bedside. Frontline clinicians (i.e., clinicians who are primarily, if not entirely, focused on patient care) carry a wealth of clinical knowledge and the lived experience of providing medical care daily. The experiences and knowledge of frontline clinicians likely differ from those of clinician researchers who spend the majority of their time away from the bedside. While there is a continuum from one role to the other, most clinicians cluster at one end of

Prior Presentations None

Received August 24, 2020

Accepted December 30, 2020

Published online February 2, 2021 this spectrum. We propose multiple ways, at various levels of investment, by which a clinician can engage and contribute to programs of scientific inquiry.

An underutilized option to contribute to clinical research is stakeholder engagement. Stakeholder engagement aligns scientific discovery with the lived context of patients and clinicians to boost the relevance and uptake of research findings. There is general agreement that engaging stakeholders in research provides added value, ${ }^{1}$ but it has largely been limited to patients and their caregivers (e.g., the Patient-Centered Outcomes Research Institute $\left(\right.$ PCORI)). ${ }^{2}$ Frontline clinicians are rarely consulted ${ }^{3}$ outside of certain pragmatic trial settings. ${ }^{4}$

\section{Why Should Frontline Clinicians Engage with Research?}

Research that ignores the complexities of health care delivery is doomed to stagnate. Research findings take an average of 17 years from publication to practice integration. ${ }^{5}$ Additionally, there is a well-recognized "voltage drop" during the translation from trials to bedside ${ }^{6}$ such that intervention effectiveness in regular practice is often notably less than efficacy in clinical trials. ${ }^{5}$ This reduction is due in part to differences in participants in a general patient population versus a tightly controlled trial, but is also likely due to limitations of intervention feasibility and inappropriate or absent implementation strategies to support adoption. ${ }^{7}$ Incorporation of clinician feedback during early stages of research (e.g., protocol development) could lead to improved success during dissemination, implementation, and, ultimately, real-world effectiveness.

Yet, many frontline clinicians have little exposure to research $^{8}$ which may explain many evidence-based practices that do not integrate easily with existing clinical workflow and resources. ${ }^{9}$ Intentional frontline clinician-researcher collaborations can improve the success of intervention implementation ${ }^{10,11}$ and expand research capacity. ${ }^{12}$ Moreover, such professional opportunities may access underutilized strengths of the clinician workforce, which is increasingly cross-trained with diverse and valuable skill sets. ${ }^{13}$ 


\section{What Could Frontline Clinicians Contribute to a Collaboration with Researchers?}

Clinicians can communicate day-to-day decisional dilemmas that can spark the development of new research questions. ${ }^{14}$ In their analytic-deliberative model of stakeholder participation, Deverka and colleagues include values and professional experience as potential inputs from stakeholders. ${ }^{15}$ Just as we seek to better understand a patient's lived experience in order to tailor a disease-specific intervention, so too should we consider adapting innovations to a clinician's lived experience of delivering care.

Contributions from frontline clinicians exist on a continuum with clinician-investigators. To provide experiential expertise, frontline clinicians do not need methodologic expertise. However, both clinical and methodologic knowledge are sometimes needed. Clinician-investigators are often well positioned to provide in-depth clinical knowledge to nonclinical $\mathrm{PhD}$ collaborators and are fluent in the language of research and clinical care with which they can provide relatable interpretation of findings or lead research studies as principle investigator themselves.

\section{Barriers to Research Engagement for Frontline Clinicians}

Multiple factors keep frontline clinicians from engaging. Most apparent is that research and patient care live by different timelines. Researchers are beholden to the rhythm of grant cycles and the peer-review process, while frontline clinicians must nimbly respond to the daily demands of patient care as well as rapid shifts in quality metrics. Moreover, as health care systems grow, it is less likely that researchers are co-located with frontline clinicians in a manner that would facilitate casual "water cooler" collaborations. Beyond logistics, a lack of common language must be overcome. Researchers need to know what to ask of frontline clinicians and at what point in the process. Frontline clinicians must feel capable of adding value to the research process. ${ }^{16}$ Last, a recent regional survey of frontline clinician experience with research found that $31.5 \%$ of frontline clinicians did not "know how to get started." 17

\section{Opportunities for Frontline Clinicians to Give Meaningful Input}

To explore meaningful possibilities for frontline clinicians to share their perspective with research activities, we use the continuum of engagement as presented by the UCSF Clinical and Translational Science Institute. ${ }^{18}$ Handley and colleagues note three levels along the engagement continuum, specifically minimal, supportive, and participatory. We describe potential approaches and context at each level of engagement (Fig. 1).

- Minimal ( $<\mathbf{1 0} \mathbf{h}$ per year): At this level, one would not significantly contribute to the development, design, or implementation of research studies ${ }^{18}$, nor have protected time for engagement. However, provision of valuable input is possible through primarily advisory opportunities, including:

Interacting with study teams around care provision: frontline clinicians practicing at an academic medical center might interact with study investigators when providing care for patients who are enrolled in clinical trials; such interactions involve little to no substantive change in normal responsibilities.

Brief, single-instance feedback interactions: Frontline clinicians might provide insightful feedback if approached by research teams with well-defined and discrete queries to inform study activities such as recruitment language.

Episodic participation in feedback sessions: frontline clinicians can engage in one-time, individual qualitative interviews, focus groups, or design-thinking workshops to provide feedback for a particular study or clinical topic. ${ }^{19}$ This type of input can directly inform future evaluations ${ }^{20}$ or iterations of particular interventions. ${ }^{19,21}$

- Supportive (10-50 h per year): With supportive engagement, frontline clinician-driven questions and priorities are incorporated into study design. ${ }^{18}$ This level requires a measurable increase in time and a longitudinal commitment. Supportive roles span more intensive advisory roles to increased engagement with iterative contributions to study design and conduct.

Practice-based research networks (PBRNs): The preexisting nature of these multi-practice infrastructures allows for rapid and timely response to research needs. Frontline clinicians participating in PRBNs have made a commitment to the concept and exercise of research engagement and can run site-level study activities, serve as a recruitment liaison, or make local stakeholder connections. Examples of primary care-based PBRNs abound and are productive contributors to research and quality improvement. $^{22-25}$

Research advisory board/clinician engagement panels: Frontline clinicians might join formal groups convened to provide direct feedback to research activities or agendas. For example, many PBRNs have a standing group with frontline clinician representation for provision of feedback to research proposals and lines of inquiry - distinct from those who are conducting the multisite studies. Similarly, the research community has seen a recent growth in standing patient engagement panels developed to function in an ongoing manner to support a single large study or a series of studies across a research center. ${ }^{26} \mathrm{~A}$ group of frontline clinicians who undergo basic training in research concepts could meet on a regular basis to review and provide input on aspects of planned and ongoing studies. ${ }^{27}$

Systematic reviews: Participating in the conduct of systematic approaches to evidence synthesis across a body of 


\section{Research Engagement for Frontline Clinicians}

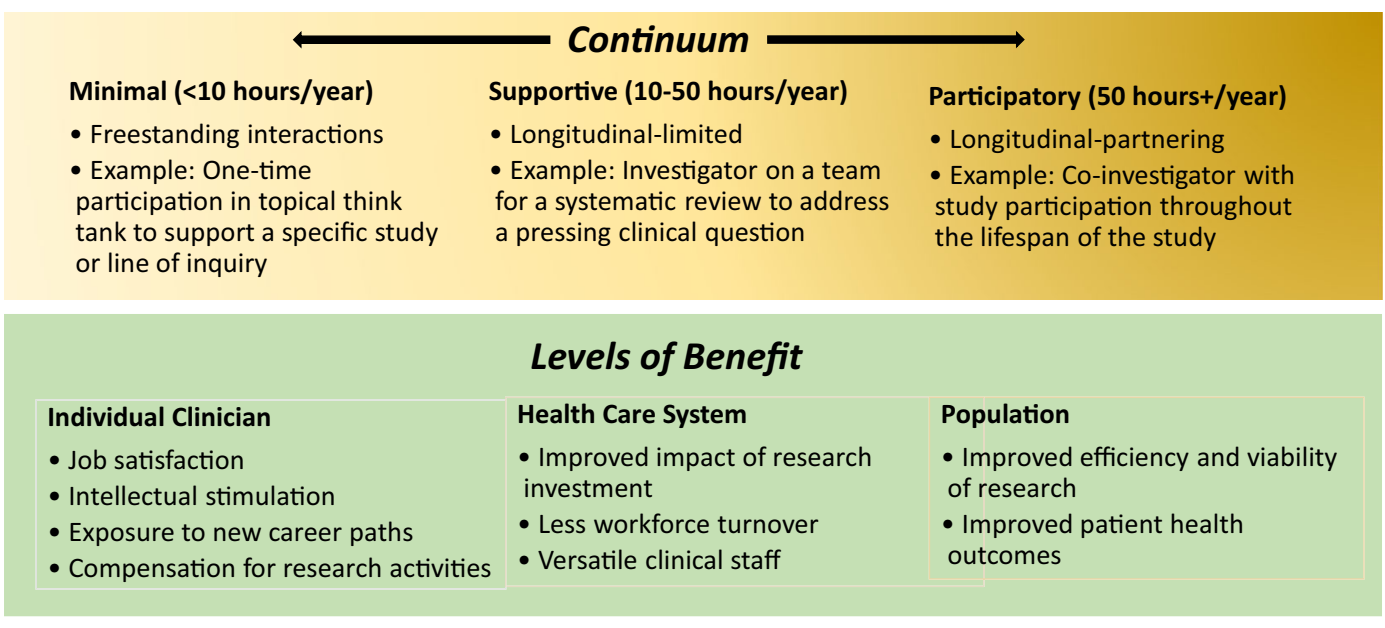

Figure 1 Continuum of opportunities for and benefits from frontline clinician engagement in research.

literature in response to a clinical question is an opportunity that does not involve human subjects or primary data collection thus allowing for regulatory flexibility in participation. In our evidence synthesis program, $^{28} 29$ we regularly seek frontline clinicians as team members to provide key clinical experience and expertise for review conduct and interpretation of results.

- Participatory (> $\mathbf{5 0}$ h per year): This highest level of engagement implies a true collaborative effort, which can develop over time and can be exemplified in community-based participatory research (CBPR). ${ }^{30,} 31$ While clinicians keep their "frontline" identity, the dedicated time and longitudinal commitment can be more substantial as they help shape research through defining research questions and study design methods and through interpretation and dissemination of results. Here, frontline clinicians are full, active members of research activities such as assisting with participant recruitment or collection of study data, and approach the clinician-investigator end of the continuum.

Co-investigator or co-principal investigator: Frontline clinicians can take on the role of a core study team member attending regular study meetings and participating throughout the lifespan of the investigation from proposal development to study execution. ${ }^{32}$

Executive committee member/stakeholder panel: Single and multisite clinical trials often have a committee of stakeholders who help oversee the operational and scientific aspects of a trial. Depending on the study, the level of involvement for a frontline clinician could be more or less (i.e., consistent with the supportive vs participatory level).

\section{What About Frontline Clinicians Practicing Outside of Academia?}

For those practicing in the community, the possibilities for connections to research may not be as visible. Approximately $46 \%$ of US clinicians provide care in private practices. ${ }^{33}$ Due to differences in health system resources and structures, clinical care delivery challenges faced in these settings are likely different from university practices. As most patients are seen outside of the academic setting, involving nonacademic practices may improve external generalizability, promote ready implementation, and expand the reach of scientific advancements. ${ }^{34}$ The following are examples of ways to involve nonacademic frontline clinicians:

- Collaborations with nonacademic research entities: A small portion of nonacademic practices and frontline clinicians participate in trials run by contract research organizations or industry.

- PBRNs: Many PBRNs partner with nonacademic practices including community-based clinics and nonacademic medical center-affiliated clinics. This arrangement is often seen in association with professional societies. ${ }^{35-37}$

\section{Proposed Metrics for Success}

How will we know if engagement of frontline clinicians has been meaningful? Lavallee and colleagues point to the importance of dual-level evaluation of stakeholder engagement, including outcomes at the process and endpoint levels. ${ }^{38}$ Process outcomes are generally measureable in the short term and are reflective of the engagement activity itself, for example, frontline clinician satisfaction with the engagement process due to a sense of legitimacy and trust in the interactions with 
research teams. Process outcomes can also measure the extent to which involvement impacts study conduct, including activities such as recruitment, retention, and intervention fidelity. Endpoint outcomes are generally longer term outcomes and the ultimate goal of a line of scientific inquiry, such as improved patient health outcomes, more sustainable interventions, or better uptake of findings. Process outcomes are likely more useful to an individual frontline clinician or study team, while endpoint outcomes may be more useful at the health care system or funding-body level.

\section{Next Steps}

Mitigating obstacles to involving frontline clinicians in research activities will require creativity and cooperation. One opportunity is the use of advances in electronic health record (EHR) systems to bridge communication between research teams and frontline clinicians. For example, a clinical trial decision support system could facilitate EHR-based recruitment from primary care providers. ${ }^{39}$ This approach could work for frontline clinicians with an academic medical center or health care system, however, community-based providers may not yet have this pathway for collaboration.

Next, building leadership support is key to the success of engaging frontline clinicians. ${ }^{40}$ In order for busy frontline clinicians to dedicate time and effort to research engagement, they need to make a convincing value proposition to their supervisors. Similarly, researchers need to budget equitable compensation for frontline clinician effort commiserate with level of engagement. ${ }^{41}$ Examples of success in allocating project funding to practice and clinician engagement can be seen with PBRNs ${ }^{41}$ and guidance on appropriate budgeting is available. ${ }^{42,}{ }^{43}$ Meanwhile, health care systems will need to develop business and compensation models that support team-based approaches to increasing efficiency to allow and incentivize the investment of clinician effort in research.

We propose that there is potential value at multiple levels (Fig. 1). At a population level, improving patient care and health outcomes is the ultimate goal of clinical practice and health services research. At a health system level, frontline clinician engagement could improve the efficiency and impact of research investments. At an individual level, collaborations could reduce clinician burnout by tapping underutilized skills or improving professional satisfaction through acknowledgement in publications and opportunities to present findings at regional or national meetings.

\section{CONCLUSION}

The intentional pursuit of pathways for frontline clinician involvement in research is an important aspect of meaningful engagement as learning health care systems evolve. Opportunities for such engagement exist along a spectrum of commitment intensity and offer potential value to health care systems at multiple levels.

Acknowledgments: We thank Liz Wing, MA, for editorial assistance.

Corresponding Author: Karen M. Goldstein, MD, MSPH; Department of Medicine, Division of General Internal Medicine, Duke University School of Medicine, Durham, NC, USA (e-mail: Karen. goldstein@duke.edu).

Funding K.M.G. was supported by VA HSR\&D Career Development Award (CDA \#13-263). In addition, this work was supported by the Durham Center of Innovation to Accelerate Discovery and Practice Transformation (ADAPT), (CIN 13-410) at the Durham VA Health Care System and by Department of Veterans Affairs, Veterans Health Administration, 421 Office of Research and Development, Quality Enhancement Research Initiative (ESP 09-010).

\section{Compliance with Ethical Standards:}

Disclaimer: The views expressed in this article are those of the authors and do not necessarily represent the views of the Department of Veterans Affairs.

Conflict of Interest: The authors have no conflicts of interest to declare.

\section{REFERENCES}

1. Hinchcliff R, Greenfield D, Braithwaite $\mathbf{J}$. Is it worth engaging in multistakeholder health services research collaborations? Reflections on key benefits, challenges and enabling mechanisms. Int $J$ Qual Health Care. 2014;26(2): 124-128.

2. Boaz A, Hanney S, Borst R, O'Shea A, Kok M. How to engage stakeholders in research: design principles to support improvement. Health Res Policy Syst. 2018;16(1):60.

3. Concannon TW, Fuster M, Saunders T, et al. A systematic review of stakeholder engagement in comparative effectiveness and patientcentered outcomes research. J Gen Intern Med. 2014;29(12):1692-701.

4. Moloney RM, Tambor ES, Tunis SR. Patient and clinician support for the learning healthcare system: recommendations for enhancing value. J Comp Eff Res. 2016;5(2):123-8.

5. Morris ZS, Wooding S, Grant $\mathbf{J}$. The answer is 17 years, what is the question: understanding time lags in translational research. J R Soc Med. 2011;104(12):510-20.

6. Eisenberg JM, Power EJ. Transforming insurance coverage into quality health care: voltage drops from potential to delivered quality. JAMA. 2000;284(16):2100-7.

7. Fischer $\mathbf{F}$, Lange $\mathbf{K}$, Klose $\mathbf{K}, \mathbf{G r e i n e r} \mathbf{W}, \mathbf{K r a e m e r} \mathbf{A}$. Barriers and strategies in guideline implementation-a scoping review. Healthcare (Basel). 2016;4(3).

8. Pager S, Holden L, Golenko $\mathbf{X}$. Motivators, enablers, and barriers to building allied health research capacity. J Multidiscip Healthc. 2012;553-9.

9. Harding KE, Porter J, Horne-Thompson A, Donley E, Taylor NF. Not enough time or a low priority? Barriers to evidence-based practice for allied health clinicians. J Contin Educ Health Prof. 2014;34(4):224-31.

10. Blevins D, Farmer MS, Edlund C, Sullivan G, Kirchner JE. Collaborative research between clinicians and researchers: a multiple case study of implementation. Implement Sci. 2010;576.

11. Stover AM, Tompkins Stricker $\mathbf{C}$, Hammelef $\mathbf{K}$, et al. Using Stakeholder Engagement to Overcome Barriers to Implementing Patientreported Outcomes (PROs) in Cancer Care Delivery: Approaches From 3 Prospective Studies. Med Care. 2019;57 Suppl 5 Suppl 1:S92-s99.

12. Slade SC, Philip K, Morris ME. Frameworks for embedding a research culture in allied health practice: a rapid review. Health Res Policy Syst. 2018;16(1):29.

13. Smith CM, Jung P. Millennials in medicine: cross-trained physicians not valued in medical marketplace. Fed Pract. 2019;36(12):574-578. 
14. Homer-Vanniasinkam S, Tsui J. The continuing challenges of translational research: clinician-scientists' perspective. Cardiol Res Pract. 2012;2012246710.

15. Deverka PA, Lavallee DC, Desai PJ, et al. Stakeholder participation in comparative effectiveness research: defining a framework for effective engagement. J Comp Eff Res. 2012;1(2):181-194.

16. Wenke R, Noble C, Weir KA, Mickan S. What influences allied health clinician participation in research in the public hospital setting: a qualitative theory-informed approach. BMJ Open. 2020;10(8):e036183.

17. Ciemins EL, Mollis BL, Brant JM, et al. Clinician engagement in research as a path toward the learning health system: A regional survey across the northwestern United States. Health Serv Manage Res. 2020;33(1):33-42.

18. Handley M, Potter M, Goldstein E. Community-Engaged Research with Community-Based Clinicians: A Resource Manual for Researchers. 2010;From the series: UCSF Clinical and Translational Science Institute (CTSI) Resource Manuals and Guides to Community-Engaged Research, P. Fleisher, ed.

19. Joosten YA, Israel TL, Williams NA, et al. Community Engagement Studios: A Structured Approach to Obtaining Meaningful Input From Stakeholders to Inform Research. Acad Med. 2015;90(12):1646-50.

20. Sandelowski M. Focus on qualitative methods: Using qualitative methods in intervention studies. Res Nurs Health. 1996;19(4):359-64.

21. Joosten YA, Israel TL, Head A, et al. Enhancing translational researchers' ability to collaborate with community stakeholders: Lessons from the Community Engagement Studio. J Clin Transl Sci. 2018;2(4):201-207.

22. Green LA, Hickner J. A short history of primary care practice-based research networks: from concept to essential research laboratories. J Am Board Fam Med. 2006;19(1): 1-10.

23. Mold JW, Peterson KA. Primary care practice-based research networks: working at the interface between research and quality improvement. Ann Fam Med. 2005;3 Suppl 1(Suppl 1):S12-20.

24. Westfall JM, Mold J, Fagnan L. Practice-based research-"Blue Highways" on the NIH roadmap. JAMA. 2007;297(4):403-6.

25. Frayne SM, Carney DV, Bastian L, et al. The VA Women's Health Practice-Based Research Network: amplifying women veterans' voices in VA research. J Gen Intern Med. 2013;28 Suppl 2:S504-9.

26. Newman SD, Andrews JO, Magwood GS, Jenkins C, Cox MJ, Williamson DC. Community advisory boards in community-based participatory research: a synthesis of best processes. Prev Chronic Dis. 2011;8(3):A70.

27. Dolor RJ, Campbell-Voytal K, Daly J, et al. Practice-based Research Network Research Good Practices (PRGPs): Summary of Recommendations. Clin Transl Sci. 2015;8(6):638-46.

28. Department of Veterans Affairs. Durham Center of Innovation to ADAPT. Available at: https://www.durham.hsrd.research.va.gov/VA_Evidence_ Synthesis_Program ESP.asp. Accessed December 9, 2020.

29. Department of Veterans Affairs. Health Services Research \& Development: Evidence Synthesis Program. Available at: https://www.hsrd. research.va.gov/publications/esp/. Accessed December 9, 2020.
30. Israel BA, Schulz AJ, Parker EA, Becker AB. Community-based participatory research: policy recommendations for promoting a partnership approach in health research. Educ Health (Abingdon). 2001;14(2):182-97.

31. Neill SJ. Developing children's nursing through action research. J Child Health Care. 1998;2(1):11-5.

32. DeBar LL, Jarvik JG, Tuzzio L, Vazquez MA. Assessing Feasibility: Establishing Close Partnerships With Participating Healthcare System Leaders and Staff. In: Rethinking Clinical Trials: A Living Textbook of Pragmatic Clinical Trials. Bethesda, MD: NIH Health Care Systems Research Collaboratory. Available at: https://rethinkingclinicaltrials. org/chapters/conduct/assessing-feasibility/establishing-close-partnerships-with-participating-healthcare-system-leaders-and-staff/. Accessed August 18, 2020.

33. American Medical Association. Online article; May 10, 2019. Employed physicians now exceed those who own their practices. Available at: https://www.ama-assn.org/about/research/employed-physicians-nowexceed-those-who-own-their-practices. Accessed August 12, 2020.

34. Green LA, Fryer GE, Jr., Yawn BP, Lanier D, Dovey SM. The ecology of medical care revisited. N Engl J Med. 2001;344(26):2021-5.

35. American Academy of Pediatrics. Pediatric research in office settings [online]. Available at: https://www.aap.org/en-us/professional-resources/Research/PROS/Pages/Pediatric-Research-in-Office-Setting. aspx. Accessed August 12, 2020.

36. American Academy of Family Physicians. AAFP national research network [online]. Available at: https://www.aafp.org/patient-care/nrn/ nrn.html. Accessed August 12, 2020.

37. Agency for Healthcare Research and Quality. PBRN registry [online]. Available at: https://pbrn.ahrq.gov/pbrn-registry/acp-quality-connect. Accessed August 12, 2020.

38. Lavallee DC, Williams CJ, Tambor ES, Deverka PA. Stakeholder engagement in comparative effectiveness research: how will we measure success? J Comp Eff Res. 2012;1(5):397-407.

39. Taft T, Weir C, Kramer H, Facelli JC. Primary care perspectives on implementation of clinical trial recruitment. J Clin Transl Sci. 2020;4(1):61-68

40. Matus J, Walker A, Mickan S. Research capacity building frameworks for allied health professionals - a systematic review. BMC Health Serv Res. 2018;18(1):716.

41. Dolor RJ, Schmit KM, Graham DG, Fox CH, Baldwin LM. Guidance for researchers developing and conducting clinical trials in practice-based research networks (PBRNs). J Am Board Fam Med. 2014;27(6):750-8.

42. PCORI. Patient-Centered Outcomes Research Institute. Compensation framework. Available at: https://www.pcori.org/document/compensation-framework. Accessed December 10, 2020.

43. DARTNet Institute. Available at: http://www.dartnet.info/FAQBudgeting. htm. Accessed December 10, 2020.

Publisher's Note: Springer Nature remains neutral with regard to jurisdictional claims in published maps and institutional affiliations. 